\title{
Change points in the hazard function of survival models
}

\section{Puntos de cambio en la función de riesgo de modelos de supervivencia}

MUÑOZ-VARGAS, Blanca Xochilt†*, JUÁREZ-HERNÁNDEZ, Bulmaro and REYES-CERVANTES, Hortensia Josefina

Benemérita Universidad Autónoma de Puebla, Facultad de Ciencias Físico Matemáticas, México.

ID $1^{\text {st }}$ Author: Blanca Xochilt, Muñoz-Vargas / ORC ID: 0000-0001-0000-0000, CVU CONACYT ID: 702397

ID $1^{\text {st }}$ Co-author: Bulmaro, Juárez-Hernández / ORC ID: 0000-0002-6260-1296, CVU CONACYT ID: 78065

ID $2^{\text {nd }}$ Co-author: Hortensia Josefina, Reyes-Cervantes / ORC ID: 0000-0002-1000-8922, CVU CONACYT ID: 161756

DOI: $10.35429 / J M Q M .2021 .9 .5 .1 .9$

Received July 10, 2021; Accepted December 30, 2021

\section{Abstract}

In this work, a chronological presentation of the main results and applications of the different investigations that analyze the problem of the change point in the hazard function of survival models is made, these include the constant hazard function by parts and Cox-type regression models with change points.

Survival analysis, Hazard function, Change points

\begin{abstract}
Resumen
En este trabajo se hace una presentación de manera cronológica de los principales resultados y aplicaciones de las diferentes investigaciones que analizan el problema del punto de cambio en la función de riesgo de modelos de supervivencia, estos incluyen a la función de riesgo constante por partes y modelos de regresión tipo Cox con puntos de cambio.
\end{abstract}

Análisis de supervivencia, Función de riesgo, Puntos de cambio

Citation: MUÑOZ-VARGAS, Blanca Xochilt, JUÁREZ-HERNÁNDEZ, Bulmaro and REYES-CERVANTES, Hortensia Josefina. Change points in the hazard function of survival models. Journal-Mathematical and Quantitative Methods. 2021. 59:1-9.

\footnotetext{
* Author Correspondence (Email: b.xochilt.munoz@gmail.com)

$\dagger$ Researcher contributing as first author.
} 


\section{Introduction}

The problem of testing and estimating the change points has attracted much attention in the literature, one of the most studied models in the change point problem is the normal model (univariate or multivariate), this is because the normal model is the most common model in practice. However, the change point problem has also been studied in models: exponential, gamma, regression, and survival, to name a few. In Survival Analysis, the simplest model is the constant hazard function, which corresponds to the exponential distribution and has been widely studied, however, in most applications this model is not the most appropriate, so it is use other distributions such as Weibull, log-normal, log-logistic, Gamma, Generalized Gamma, etc. for better settings. Another alternative to have better adjustments was to introduce a change point to the hazard function. The first hazard function that was studied with a change point was the constant hazard function, that is, the hazard is equal to a constant until a certain unknown moment from which the hazard is equal to another constant. This model is different from the one studied by (Hinkley, 1970) who considered a change point in a sequence of random variables.

Next, different studies of change points in the hazard function of survival models are presented, in some cases the original notation is changed to have homogeneity of notation. This work is organized as follows, in section 2 survival models with a constant hazard function by parts are presented, in section 3 some models with a non-constant hazard function with change points are shown and Cox-type regression models with change points are presented in section 4. Finally, section 5 presents the conclusions.

\section{Constant hazard function by parts}

One of the first studies that includes a change point in the hazard function of a survival model is that of (Matthews \& Farewell, 1982) who studied the time-to-failure model with constant hazard function by parts with a change point, so the hazard function is given by

$h(t)=\left\{\begin{aligned} \lambda_{1}, & \text { si } t \leq \tau \\ \rho \lambda_{1}, & \text { si } \tau<t\end{aligned}\right.$ (function $\lambda(\cdot)$ of equation (1) of (Matthews \& Farewell, 1982)) and the parameters $\lambda_{1}>0, \rho>$ 0 and $\tau>0$ have to be estimated. The parameter $\tau$ is called the change point. Since the asymptotic likelihood standard inference cannot be applied, they cited a numerical method to calculate maximum likelihood estimators, they simulated the distribution of the likelihood ratio statistic for the null hypothesis of the hypothesis set

$H_{0}: \rho=1 \& \tau=0$ vs $H_{1}: \rho \neq 1 \& \tau>0$,

and they applied this model to data from patients with acute non-lymphoblastic leukemia. As a result, they obtained that, for uncensored time samples, certain percentiles of the simulated null distribution of the likelihood ratio statistic, $2 \widehat{\Lambda}_{0}$, are close to the percentiles of the distribution $\chi_{(2)}^{2}$, which would be the distribution asymptotic of $2 \Lambda_{0}$ if the asymptotic likelihood ratio theory could be applied. Also, they found that the procedures for uncensored data can be applied to censored data.

Later, (Nguyen, Rogers \& Walker, 1984) also studied the survival model with a constant hazard function by parts with a change point expressing the hazard function as

$h(t)=a I(0 \leq t \leq \tau)+b I(\tau<t)$,

where $I(A)$ is the indicator function of the set $A$. In this case the parameters $a>0, b>0$ and $\tau>$ 0 have to be estimated, the latter being the change point parameter. They considered the density function as a combination of two exponential densities (one truncated to the right in $\tau$ and the other lagged in $\tau$ ) with unknown weights that depend on the parameters to be estimated and showed that the log likelihood function, $l(\cdot)$, is unbounded when $b=1 /\left(\mathrm{t}_{n}-\right.$ $\tau)$ and $\tau$ is close to $t_{n}$, where $t_{n}$ is the longest observed lifetime and $n$ is the sample size, and if $a>b, l(\cdot)$ is bounded. Furthermore, for $\tau$ known (Nguyen, Rogers \& Walker, 1984) calculated the maximum likelihood estimators of the reciprocals of $a$ and $b$, denoted by $A_{n}(\tau)$ and $B_{n}(\tau)$, respectively, which depend of $\tau$. It can be verified that these expressions are recursive in terms of $n$. While for unknown $\tau$, they obtained a strongly consistent estimator of $\tau, \hat{\tau}_{n}$, examining a stochastic process with which $A_{n}\left(\hat{\tau}_{n}\right) \rightarrow 1 / a$ and $B_{n}\left(\hat{\tau}_{n}\right) \rightarrow 1 / b$ both with probability 1 . Finally, they showed the behavior of the estimators via simulation. 
Matthews \& Farewell (1985) eliminated the singularity identified by (Nguyen, Rogers \& Walker, 1984) in the $\log$-likelihood function for the constant hazard rate model by parts with a change point (1) adequately defining the likelihood function as

$L(a, b, \tau)=\left[\prod_{i=1}^{n-1}\left\{e^{-a t_{i}}\left(e^{a \epsilon}-e^{-a \epsilon}\right)\right\}\right]$

$\times\left\{e^{-a\left(t_{n}-\epsilon\right)}-e^{-a \tau-b\left(t_{n}+\epsilon-\tau\right)}\right\}$,

when $t_{n}-\epsilon<\tau<t_{n}+\epsilon, t_{1}<\cdots<t_{n}$ are the order statistics of the random sample and $n>1$ is any sample size. The Equation (2) is due to the fact that the product of the values of the probability density function in the observations must be considered as an approximation to the true probability, then the contribution to the likelihood corresponding to an observed value $t_{i}$ is proportional to $F\left(t_{i}+\epsilon\right)-F\left(t_{i}-\epsilon\right)$, i.e., the probability that the observation $t_{i}$ belongs to a small interval, that probability is finite for $i=$ $1, \ldots n$.

Matthews, Farewell \& Pyke (1985) studied the problem of testing a constant hazard rate against a constant hazard rate by parts that involves a single change point in which the hazard rate is reduced. They expressed the hazard function as

$h(t)=\left\{\begin{aligned} \lambda, & \text { si } 0 \leq t<\lambda \\ (1-\xi) \lambda, & \text { si } \tau \leq t\end{aligned}\right.$

(function $\lambda(\cdot)$ of equation (1) of (Matthews, Farewell \& Pyke, 1985)) where $0 \leq \xi<1, \lambda\rangle$ 0 and $\tau \geq 0$. Thus, $\tau$ is a change point parameter for the hazard rate and the parameters to be estimated are $\lambda, \xi$ and $\tau$. They showed that the asymptotic significance level for the hypothesis test

$H_{0}: \xi=0$ vs $H_{1}: \xi \neq 0$,

based on the maximum score statistics involves the solution to the problem of the first step time for an Ornstein-Uhlenbeck process, for this it is assumed that $\tau \in\left[\tau_{l}, \tau_{u}\right]$ with $\tau_{l}$ and $\tau_{u}$ known. Finally, they applied their results to data from non-Hodgkin lymphoma patients.
Yao (1986) considered the model (1) and eliminated the problem of the unbounded logarithmic likelihood function by proposing the constraint $\tau \leq t_{(n-1)}$ where $t_{(n-1)}$ is the $(n-$ 1)-th statistic of order of observed lifetimes. It was calculated the restricted maximum likelihood estimators for $\tau, a$ and $b$, denoted by $\hat{\tau}$, $\hat{a}$ and $\hat{b}$ respectively, showed that $\hat{\tau}$ is consistent, derived the limit distributions of $\hat{\tau}, \hat{a}$ and $\hat{b}$ and showed that $\hat{\tau}, \hat{a}$ and $\hat{b}$ are asymptotically independent.

The model (3) was also studied by (Yao, 1987), he proposed a test to contrast the null hypothesis of a constant hazard function against the alternative of a change point in the constant hazard function by parts (hypothesis set (4)). It was showed that this problem is related to a quality control problem (mentioned as Problem B), due to this relationship he proposed a statistical test for (4) that is based on a test with a Bayesian approach to Problem B. The main advantages of this test are its computational simplicity and the availability of the theory of the distribution of small and large samples.

Worsley (1988) also studied the hypothesis set of constant hazard function against a change point in the constant hazard rate by parts at an unspecified time. The author stated the hazard function as follows

$h(t)= \begin{cases}\lambda_{1}, & \text { si } t \leq \tau \\ \lambda_{2}, & \text { si } \tau<t,\end{cases}$

(function $\lambda(\cdot)$ of the first equation of (Worsley, 1988)) where $\lambda_{1}>0$ and $\lambda_{2}>0$ are the hazard rates and $\tau>0$ is the change point. Since the likelihood ratio is unbounded, the author provided alternatives for eliminating the singularity (in which the change point is assumed to belong to an interval and in artificially censoring the largest observation) and he found the exact null distribution of a statistic of restricted likelihood ratio test.

This distribution is not affected by type II censorship but strongly depends on the interval in which the change point is assumed to belong. He also compared exact percentage points with simulated percentage points reported in the literature. Finally, he applied his results to data from patients with acute non-lifoblastic leukemia that (Matthews \& Farewell, 1982) had previously studied. 
Chang, Chen \& Hsiung (1994) considered hazard rate models with a change point that allows random censorship in which

$$
h(t)=\beta+\theta I(\tau \leq t)
$$

(function $\lambda(\cdot)$ of equation (1.1) of (Chang, Chen $\&$ Hsiung, 1994)) where $\beta>0, \beta+\theta>0$ and $\tau>0$ is the parameter of change point. They proposed a change point estimator by examining a Nelson-Aalen type functional estimator in the context of counting processes and established the consistency and asymptotic distribution of the proposed estimator.

Chen \& Baron (2014) studied the model with a constant hazard function $h(t)=\lambda_{0}$ until an unknown time $\tau$, the change occurs in time $\tau$ and $h(\cdot)$ changes to a new value $\lambda_{1}$ and it stays that way thereafter. Therefore,

$h(t)=\lambda_{0} I(t \leq \tau)+\lambda_{1} I(\tau<t)$,

(function $\lambda(\cdot)$ of equation (1) of (Chen $\&$ Baron, 2014)) where $\lambda_{0}, \lambda_{1}>0$ and $\tau>0$ is the change point, the main parameter of interest. They proposed a new alternative estimation procedure based on the Kaplan-Meier estimation of the survival function followed by the least square estimation of the change point and established strong consistency of all estimators. The authors applied their methods to data from a clinical trial of the strong-drug treatment program.

Goodman, Li \& Tiwari (2011) studied the following survival model whose hazard function is constant in parts

$h(t)=\left\{\begin{array}{c}\alpha_{1}, \text { si } 0 \leq t \leq \tau_{1}, \\ \alpha_{2}, \text { si } \tau_{1}<t \leq \tau_{2}, \\ \vdots \\ \alpha_{k+1}, \text { si } \tau_{k}<t,\end{array}\right.$ (function $\lambda(\cdot)$ of equation (1) of (Goodman, $\mathrm{Li}$ \& Tiwari, 2011)) where $0=\tau_{0}<\tau_{1}<\cdots<$ $\tau_{k}<\infty$ are the change points, $k$ the number of change points in the model and $\alpha_{j}$ the value of the hazard function between the times $\tau_{j-1}$ and $\tau_{j}$. They proposed a maximum likelihood estimate by profiles and based on the independence of the estimates of $\alpha_{j}$ and $\tau_{j}$ that (Yao, 1986) showed, they proposed a Wald-type test statistic only for the parameters of the hazard rate $\left(\alpha_{j}\right)$ while treating the parameters of the change point $\left(\tau_{j}\right)$ as fixed, that is, the set of hypotheses that test is

$$
H_{0}: \alpha_{k-1}-\alpha_{k}=0 \text { vs } H_{1}: \alpha_{k-1}-\alpha_{k} \neq 0 .
$$

In addition, they proposed a model selection algorithm that uses sequential tests for the constant hazard model by parts, this method is based on the data and allows estimating not only the number of change points in the hazard function, but also where they occur those changes and developed an alpha spending scheme such that the maximum number of change points, $k$, need not be prespecified. Finally, they conducted simulation studies that confirm the validity of the proposed test and applied their methods to test the change points in the hazard rates of prostate cancer patients.

Majumder \& Mitra (2019) proposed a test to detect trend changes in hazard functions. The testing problem is $H_{0}$ : constant failure rate vs $H_{1}$ : bathtub shaped failure rate. Test statistics based on a weighted integral approach are constructed using a measure of deviation from exponentiality. They exploit the theory of the Lstatistic to obtain the exact and asymptotic distributions of their statistics and establish the consistency of the test. Finally, a simulation study and applications to real-life data sets are presented to illustrate their results.

\section{Non-constant hazard function with a change point}

In many applications, the hazard function is not constant before or after the change point, so a model is needed that encompasses other possibilities. Wu, Zhao \& Wu (2003) extended the model with a constant hazard function with a change point and studied the model with a hazard function given by 
$h(t)=[\beta+\theta I(t>\tau)] \lambda_{0}(t ; \underline{\gamma})$,

(function $\lambda(\cdot)$ of equation (1.2) of (Wu, Zhao \& $\mathrm{Wu}, 2003))$ where $\lambda_{0}(\because \underline{\gamma})$ is a continuous baseline hazard function that depends on an unknown parameter vector $\underline{\gamma}$. The model (5) covers many important models commonly used in Survival Analysis, such as the models exponential $\quad\left(\lambda_{0}(x ; \underline{\gamma})=1\right), \quad$ Weibull $\left(\lambda_{0}(x ; \underline{\gamma})=x^{\gamma}\right)$, extreme $\left(\lambda_{0}(x ; \underline{\gamma})=e^{\gamma x}\right)$ and log-logistics $\left(\lambda_{0}(x ; \underline{\gamma})=x^{\gamma_{1}} /\left(1+\gamma_{2} x^{\gamma_{1}+1}\right)\right)$. Considering a random sample with random censorship, the authors proposed a nonparametric estimator of the change point in the context of counting processes. In addition, they showed that both the change point estimator and the other estimators are consistent.

Brazzale, et al. (2019) studied the survival model with a decreasing hazard rate before the change point and a constant hazard rate after the change point given by

$h(t)=h_{1}(t) I(t<\tau)+\lambda I(t \geq \tau)$,

where $\lambda>0, \tau \geq 0$ is the change point, $h_{1}(\cdot)$ is a continuous hazard function before the change point and the hazard function is also continuous at the change point, that is, $h_{1}(\tau)=\lambda$. They presented a new method for estimating the change point that is based on fitting a regression to the $p$ values of the hazard rate tests in small time intervals. In the end, they presented three examples of real data that describe the survival patterns of seriously ill patients, whose mortality rates persist beyond hospital discharge.

\section{Cox-type regression models with change points}

The proportional hazard or Cox model is used to study the relationship between survival time and covariates, which can be constant or timedependent, and can be studied using parametric and semi-parametric methods. The semiparametric proportional hazards model proposed by (Cox \& Oakes, 1984) has been widely adopted in clinical trials with time-to-event results to compare an experimental versus standard care treatment. A key assumption in the Cox model is that the hazard ratio function is a constant over time, which is often violated, as there will be a lag period before the experimental treatment reaches its full effect. For example, the KaplanMeier estimates of survival curves in many oncology clinical trials with two treatment arms overlap at the start of the treatment period up to a certain time point and then begin to separate, indicating that the constant hazard ratio assumption may have been violated and there may be a change point in the hazard ratio function (He, Fang \& Su, 2012).

The semiparametric transformation models have been proposed as a broad class of regression models and includes the proportional hazards and proportional odds models as special cases. The other useful and flexible alternative is the Aalen's additive risk model, which allows the influence of covariates on a hazard function to vary separately and nonparametrically through time. Although allowing greater flexibility than the proportional hazards model, the number of covariates that can be handled by Aalen's model is quite limited. To reduce the number of functions needed to be estimated, McKeagues \& Sasieni (1994) introduced a partly parametric version of Aalen's model, called the partly Aalen's additive hazards model, where the influence of some covariates varies nonparametrically over time and that of the remaining covariates is restricted to be constant in time (Shen, 2020).

Other extensions of the Cox model have been investigated in the literature, for example, the two-step regression model studied by (Anderson \& Senthilselvan, 1982)

$h(t, \underline{z})=\left\{\begin{array}{l}\lambda_{0}(t) \exp \left(\underline{\alpha}^{\prime} \underline{z}\right), \text { si } t \leq B \\ \lambda_{1}(t) \exp \left(\underline{\gamma}^{\prime} \underline{z}\right), \text { si } B<t\end{array}\right.$

(function $\lambda(\cdot)$ of equation (3) of (Anderson \& Senthilselvan, 1982)) where $\lambda_{0}(\cdot)$ and $\lambda_{1}(\cdot)$ are unknown functions, $\underline{Z}$ is a vector of covariates, $\underline{\alpha}$ and $\underline{\gamma}$ refer to the dependence of the hazard function in $\underline{z}$ to short and long term, respectively, and $B$ is a change point. Other extensions to the proportional hazard model that also include change points in the hazard function are presented below. model

Liang, Self \& Liu (1990) considered the

$\mathrm{h}(\mathrm{t})=\lambda_{0}(\mathrm{t}) \exp \left\{(\beta+\theta \mathrm{I}(\mathrm{t} \leq \tau)) \mathrm{Z}+\underline{\gamma}^{\prime} \underline{X}\right\}$ 
(function $\lambda(\cdot)$ of equation (1) of (Liang, Self $\&$ Liu, 1990)) where $\lambda_{0}(\cdot)$ is the underlying hazard rate, $Z$ is a variable believed to be related, possibly with different magnitudes, to the survival time, $X$ is a vector of covariates whose effects do not vary in time and $\tau$ is a change point in time that belongs to the interval $[a, b]$ with known $a$ and $b$. They proposed a maximum score test statistic for the hypothesis game

$H_{0}: \theta=0$ vs $H_{1}: \theta \neq 0$,

and applied their results to a data set from a longitudinal study of hypertension. model

Luo \& Boyett (1997) studied the following

$$
h(t)=\lambda_{0}(t) \exp \left\{\beta_{0} I\left(X \leq \theta_{0}\right)+\underline{\alpha}_{0}^{\prime} \underline{Z}\right\}
$$

(function $\lambda(\cdot)$ of equation (1) of (Luo \& Boyett, 1997)) where $X$ is a one-dimensional covariate and $\mathrm{Z}$ is $p$-dimensional, in this model a constant is added after reaching a threshold. The parameters to be estimated are $\theta_{0}, \beta_{0}$ and $\underline{\alpha}_{0}$. They test consistency of partial maximum likelihood estimators. Finally, they applied the above method to a cohort of patients with lineage B leukemia treated at St. Jude Children's Research Hospital.

Luo, Chen \& Boyett (1996) also studied the model (6), presented asymptotic distributions for maximum likelihood estimators, and applied their results to simulated data and data of patients treated for newly diagnosed acute lymphoblastic leukemia.

Pons (2003) introduced the model

$h(t)=\lambda_{0}(t) \exp \left\{\underline{\alpha}^{\prime} \underline{Z}_{1}(t)+\underline{\beta}^{\prime} \underline{Z}_{2}(t) I\left(Z_{3} \leq \zeta\right)+\underline{\gamma}^{\prime} \underline{Z}_{2}(t) I\left(Z_{3}>\zeta\right)\right\}$,

(function $\lambda(\cdot)$ of equation (1.1) of (Pons, 2003)) where the influence of a covariate jumps in a certain limit $\zeta$. In this case, the author showed that the estimator of the change point parameter is $n$-consistent, the estimators of the regression parameters are $\mathrm{n}^{1 / 2}$-consistent and established the asymptotic distributions of the estimators.

He, Fang \& Su (2012) studied the following change point model

$$
\beta(t)=\left\{\begin{array}{c}
\beta_{1}, \text { si } 0 \leq t \leq \tau_{1}, \\
\beta_{2}, \text { si } \tau_{1}<t \leq \tau_{2}, \\
\vdots \\
\beta_{k+1}, \text { si } \tau_{k}<t
\end{array}\right.
$$

where $\beta(\cdot)$ is the logarithmic function of the hazard ratio, $0=\tau_{0}<\tau_{1}<\cdots<\tau_{\mathrm{k}}<\infty$ are the change points, $k$ is the number of change points in the model and $\beta_{j}$ is the value of the logarithm of the hazard ratio between the time points $\tau_{j-1}$ and $\tau_{j}$. They proposed maximizing the partial log likelihood function to obtain the estimators and a sequential test procedure to determine the number of change points in the logarithmic function of the hazard ratio.

Dupuy (2006) studied the following hazard model with a change point:

$$
\begin{aligned}
& \mathrm{h}(\mathrm{t} \mid \underline{\mathrm{Z}})=(\alpha+\theta \mathrm{I}(\mathrm{t}>\tau)) \\
& \times \exp \left\{(\underline{\beta}+\underline{\gamma} \mathrm{I}(\mathrm{t}>\tau))^{\prime} \underline{\mathrm{Z}}\right\}
\end{aligned}
$$

(function $\lambda(\cdot)$ of equation (3) of (Dupuy, 2006)) where $\alpha>0, \alpha+\theta>0$ and $\tau$ is an unknown change point in time; furthermore, $\underline{\beta}$ and $\underline{\gamma}$ are unknown regression coefficients. $\mathrm{He}$ also considered the following hazard function, which allows the covariates to be time dependent, which often appear in the survival analysis literature:

$$
\begin{aligned}
& h(t \mid \underline{Z})=(\alpha+\theta I(t>\tau)) \\
& \times \exp \left\{(\underline{\beta}+\underline{\gamma} I(t>\tau))^{\prime} \underline{Z}(t) .\right.
\end{aligned}
$$

It was obtained consistent estimators for the models (7) and (8). He showed that under some established conditions, the estimators converge in probability, this is tested by the modern empirical process theory.

Later, Palmeros, Tajonar \& Juárez (2011) developed the case of time-dependent covariates since (Dupuy, 2006) only mentions that should be considered to carry out the demonstrations in this case.

Palmeros (2012) proposed the following combined parametric model:

$$
\mathrm{h}(\mathrm{t} \mid \mathrm{Z}, \underline{\mathrm{X}})=(\alpha+\theta \mathrm{I}(\mathrm{t}>\tau)) \lambda \zeta(\lambda \mathrm{t})^{\zeta-1} \exp \left\{[\beta+\gamma \mathrm{I}(\mathrm{t}>\tau)] \mathrm{Z}+\underline{\rho^{\prime}} \underline{X}\right\},
$$


where $\alpha>0, \alpha+\theta>0, \lambda>0, \zeta>0$, $\tau$ is the change point parameter, $\beta \in \mathbb{R}, \gamma \in \mathbb{R}-\{0\}$ and $\rho \in \mathbb{R}^{q}$ is the regression coefficient, $Z$ is the covariate in charge of recording a treatment and $\underline{X} \in \mathbb{R}^{q}$ is a vector of other risk factors associated with the event of interest. It was developed the estimation process and demonstrated the consistency of the proposed estimators.

Gandy, Jensen \& Lütkebohmert (2005) studied the following variant of the Cox model with a smooth change point at an unknown limit $h_{i}(t, \underline{\theta})=\lambda_{0}(t) R_{i}(t) \exp \left\{\underline{\beta}_{1}^{\prime} \underline{Z}_{1 i}(t)+\beta_{2} Z_{2 i}+\beta_{3}\left(Z_{2 i}-\xi\right)^{+}\right\}$, (function $\lambda_{i}(\cdot)$ of (Gandy, Jensen \& Lütkebohmert, 2005)) where $\underline{\theta}=\left(\xi, \underline{\beta}^{\prime}\right)^{\prime}$ with $\underline{\beta}=\left(\underline{\beta}_{1}^{\prime}, \beta_{2}, \beta_{3}\right)^{\prime} \in \mathrm{B} \subset \mathbb{R}^{p+2}$ is the vector of the regression parameters and $\lambda_{0}(\cdot)$ is the baseline hazard function and $R_{i}(\cdot)$ is a process that only takes values 1 or 0 indicating whether an individual is at risk or not. The change point is indicated by $\xi$, which is a parameter that belongs to a compact interval $\left[\xi_{1}, \xi_{2}\right]$ of known parameters $\xi_{1}$ and $\xi_{2}$. It is assumed that the true value of the parameter, $\underline{\theta}_{0}=\left(\xi_{0}, \underline{\beta}_{0}^{\prime}\right)^{\prime}$, where $\underline{\beta}_{0}=\left(\underline{\beta}_{10}^{\prime}, \beta_{20}, \beta_{30}\right)^{\prime}$, is identifiable, with $\beta_{30} \neq$ $\overline{0}$. In this model $\underline{\theta}_{0}$ is estimated by the value $\underline{\theta}_{n}$ that maximizes the logarithm of the partial likelihood. Finally, The authors showed that under some conditions there exists a neighborhood $\Theta_{0}$ of $\underline{\theta}_{0}$ such that if $\underline{\hat{\theta}}_{n}$ belongs to $\Theta_{0}$, it follows that $\underline{\hat{\theta}}_{n}$ converges in probability to $\underline{\theta}_{0}$ when $n \rightarrow \infty$.

The model proposed by (Jensen \& Lütkebohmert, 2008) is an additional extension of the one suggested in (Gandy, Jensen \& Lütkebohmert, 2005). Now more than a single change point is allowed to be in the model, the covariates are time dependent, and the counting process can jump more than once. The model involves $m$ change points and $p$ ordinary covariates (without change points), and is given as follows

$h_{i}(t, \underline{\theta})=\lambda_{0}(t) R_{i}(t) \exp \left\{\underline{\beta}_{1}^{\prime} \underline{Z}_{1 i}(t)+\underline{\beta}_{2}^{\prime} \underline{Z}_{2 i}(t)+\underline{\beta}_{3}^{\prime}\left(\underline{Z}_{2 i}(t)-\underline{\xi}\right)^{+}\right\}$, (function $\lambda_{i}(\cdot)$ of equation (1) of (Jensen \& Lütkebohmert, 2008)) where $\underline{\theta}=\left(\underline{\xi}^{\prime}, \underline{\beta^{\prime}}\right)^{\prime}$ with $\underline{\xi} \in \Xi \subset \mathbb{R}^{m} \quad$ and $\quad \underline{\beta}=\left(\underline{\beta}_{1}^{\prime}, \underline{\beta}_{2}^{\prime}, \underline{\beta}_{3}^{\prime}\right)^{\prime} \in B \subset$ $\mathbb{R}^{p+2 m}$. Here $\underline{\xi}$ and $\underline{\beta}$ are the vectors of change points and regression parameters, respectively, $\lambda_{0}(\cdot)$ is the basal intensity and $R_{i}(\cdot)$ is a process that takes only the values 1 and 0 to indicate if the subject is at risk or not. For brevity, it is considered

$\lambda_{i}(t, \theta)=\lambda_{0}(t) R_{i}(t) \exp \left\{\underline{\beta^{\prime}} \underline{\tilde{Z}}_{i}(t ; \underline{\xi})\right\}, \quad$ where $\underline{\tilde{Z}}_{i}(t ; \underline{\xi})=\left(\underline{Z}_{1 i}^{\prime}(t), \underline{Z}_{2 i}^{\prime}(t),\left(\left(\underline{Z}_{2 i}(t)-\underline{\xi}\right)^{+}\right)^{\prime}\right)^{\prime}$.

It is assumed that the vector of change points belongs to a known rectangle $\Xi:=\left[\xi_{11}, \xi_{21}\right] \times$ $\left[\xi_{12}, \xi_{22}\right] \times \ldots \times\left[\xi_{1 m}, \xi_{2 m}\right]$. The assumption that the values $\xi_{11}, \xi_{21}, \xi_{12}, \xi_{22}, \ldots, \xi_{1 m}, \xi_{2 m}$ are known is not a big restriction in real applications. The fundamental parameter $\underline{\theta}_{0}$ of this Cox-type model can be estimated with the value $\underline{\hat{\theta}}_{n}$ that maximizes the logarithm of the partial likelihood. It was showed that the estimators of the regression parameters and of the change points are $\sqrt{n}$-consistent, they demonstrated asymptotic normality and applied their model to three sets of data: actuarial, primary biliary cirrhosis (PBC) and electric motors.

\section{Conclusions}

The problem of the point of change has been studied in different types of models, in particular, in survival models. The first survival model with a change point that was studied was the one that has a constant hazard function in parts with a change point, in this case the loglikelihood function is unbounded when the change point is close to the larger observed time, so different studies were carried out with alternatives to eliminate this problem and later the model with several change points was studied. Since in most cases constant hazard is not adequate, change points were also included in other types of hazars functions, among which are those corresponding to Cox-type regression models, by introducing change points in these models defined different hazard functions since the point of change can occur at an unknown time or depend on a covariate. Another alternative that has been studied is the continuous hazard function with a change point, since a sudden change is not very realistic for most applications. 
As a result of all these studies, there is a variety of survival models with change points, in the majority, the estimation is based on the likelihood function, although recently other options have been explored and algorithms have been proposed that allow the identification of more than one change point.

\section{Acknowledgements}

The authors are grateful to all anonymous reviewers for their comments on an earlier version of this manuscript which have led to an improvement in the presentation of the paper.

\section{References}

Anderson, J. A. \& Senthilselvan, A. (1982). A two-step regression model for hazard functions. Journal of the Royal Statistical Society, 31(1). Pp: 44-51.

Brazzale, A. R.; Küchenhoff, H.; Krügel, S.; Schiergens, T. S.; Trentzsch, H. \& Hartl, W. (2019). Nonparametric change point estimation for survival distributions with a partially constant hazard rate. Lifetime Data Anal, 25. Pp: 301-321.

Chang, I. S.; Chen, C. H. \& Hsiung, C. A. (1994). Estimation in change-point hazard rate models with random censorship. Change-point problems, 23. Pp: 78-92.

Chen, X. \& Baron, M. (2014). Change-Point Analysis of Survival Data with Application in Clinical Trials. Open Journal of Statistics, 4. Pp: 663-677.

Cox, D. R. \& Oakes, D., (1984). Analysis of Survival Data. 1st ed., Chapman \& Hall, Gran Bretaña.

Dupuy, J. F. (2006). Estimation in a changepoint hazard regression model. Statistics \& Probability Letters, 76. Pp: 182-190.

Gandy, A.; Jensen, U. \& Lütkebohmert, C. (2005). A Cox model with a change-point applied to an actuarial problem. Brazilian Journal of Probability and Statistics, 19. Pp: 93109.
Goodman, M. S.; Li, Y. \& Tiwari, R. C. (2011). Detecting multiple change points in piecewise constant hazard functions. Journal of Applied Statistics, 38(11). Pp: 2523-2532.

He, P.; Fang, L. \& Su, Z. (2012). A sequential testing approach to detecting multiple change points in the proportional hazards model. Statistics in medicine, 32(7). Pp: 1239-1245.

Hinkley, D. V. (1970). Inference about the Change-Point in a sequence of random variables. Biometrika, 57(1). Pp: 1-17.

Jensen, U. \& Lütkebohmert, C. (2008). A Coxtype regression model with change-points in the covariates. Lifetime Data Anal, 14. Pp: 267-285.

Liang, K.; Self, S. \& Liu, X. (1990). The Cox proportional hazard model with change-point: An epidemiologic application. Biometrics, 46(3). Pp: 783-793.

Luo, X. \& Boyett, J. (1997). Estimations of a threshold parameter in Cox regression. Communications in Statistics-Theory and Methods, 26(10). Pp: 2329-2346.

Luo, X.; Chen, G. \& Boyett J. M. (1996). Application of Cox regression with a change point in clinical studies. In: Jewell N.P., Kimber A.C., Lee ML.T., Whitmore G.A. (eds). Lifetime Data: Models in Reliability and Survival Analysis. Springer, Boston, MA. Pp: 213-217.

Majumder, P., \& Mitra, M. (2019). Detecting trend change in hazard functions-an L-statistic approach. Statistical Papers, 62(1). Pp: 31-52.

Matthews, D. E. \& Farewell, V. T. (1982). On testing for a constant hazard against a changepoint alternative. Biometrics, 38(2). Pp: 463468 .

Matthews, D. E. \& Farewell, V. T. (1985). On a singularity in the likelihood for a change-point hazard rate model. Biometrika, 71(3). Pp: 703704.

Matthews, D. E.; Farewell, V. T. \& Pyke, R. (1985). Asymptotic score-statistic processes and tests for constant hazard against a change-point alternative. The Annals of Statistics, 13(2). Pp: 583-591. 
McKeagues, I. W. \& Sasieni, P. D. (1994). A partly parametric additive risk model. Biometrika (81). Pp: 501-514.

Nguyen, H. T.; Rogers, G. S. \& Walker, E. A. (1984). Estimation in change-point hazard rate models. Biometrika, 71(2). Pp: 299-304.

Palmeros, O., (2012). Estimación en el modelo de riesgo proporcional de Cox con un punto de cambio mediante regresión Weibull. Tesis de Doctorado, Facultad de Ciencias Físico Matemáticas, Benemérita Universidad Autónoma de Puebla.

Palmeros, O.; Tajonar, F. S. \& Juárez, B. (2011). Modelo de riesgo con un punto de cambio y covariables dependientes del tiempo. Revista Investigación Operacional, 32. Pp: 114-122.

Pons, O. (2003). Estimation in a Cox regression model with a change-point according to a threshold in a covariate. The Annals of Statistics, 31(2). Pp: 442-463.

Shen, P. S. (2020). Tests for equivalence of two survival functions: alternatives to the $\mathrm{PH}$ and $\mathrm{PO}$ models. Journal of Biopharmaceutical Statistics, 31(1). Pp: 79-90.

Worsley, K. J. (1988). Exact percentage points of the likelihood-ratio test for a change-point hazard-rate model. Biometrics, 44(1). Pp: 259263.

Wu, C. Q.; Zhao, L. C. \& Wu, Y. H. (2003). Estimation in change-point hazard function models. Statistics \& Probability Letters, 63. Pp: 41-48.

Yao, Y. C. (1986). Maximum likelihood estimation in hazard rate models with a changepoint. Communications in Statistics-Theory and Methods, 15(8). Pp: 2455-2466.

Yao, Y. C. (1987). A note on testing for constant hazard against a change-point alternative. Annals of the Institute of Statistical Mathematics, 39, Part A. Pp: 377-383. 